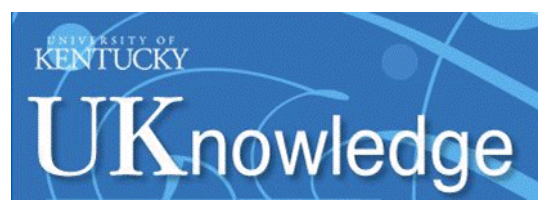

University of Kentucky

UKnowledge

$12-18-2014$

\title{
Bovine Colostrum Supplementation Optimises Earnings, Performance and Recovery in Racing Thoroughbreds
}

\author{
C. K. Fenger
}

Equine Integrated Medicine

Thomas Tobin

University of Kentucky, ttobin@uky.edu

P. J. Casey

University of Aukland, New Zealand

Edward A. Roualdes

University of Kentucky, edward.roualdes@uky.edu

\section{J. L. Langemeier \\ Equine Integrated Medicine}

See next page for additional authors

Follow this and additional works at: https://uknowledge.uky.edu/gerc_facpub

Part of the Exercise Physiology Commons, and the Large or Food Animal and Equine Medicine

\section{Commons}

Right click to open a feedback form in a new tab to let us know how this document benefits you.

\section{Repository Citation}

Fenger, C. K.; Tobin, Thomas; Casey, P. J.; Roualdes, Edward A.; Langemeier, J. L.; and Haines, D. M., "Bovine Colostrum Supplementation Optimises Earnings, Performance and Recovery in Racing Thoroughbreds" (2014). Maxwell H. Gluck Equine Research Center Faculty Publications. 29. https://uknowledge.uky.edu/gerc_facpub/29

This Article is brought to you for free and open access by the Maxwell H. Gluck Equine Research Center at UKnowledge. It has been accepted for inclusion in Maxwell H. Gluck Equine Research Center Faculty Publications by an authorized administrator of UKnowledge. For more information, please contact UKnowledge@lsv.uky.edu. 
Bovine Colostrum Supplementation Optimises Earnings, Performance and Recovery in Racing Thoroughbreds

\section{Digital Object Identifier (DOI)}

https://doi.org/10.3920/CEP140023

\section{Notes/Citation Information}

Published in Comparative Exercise Physiology, v. 10, no. 4, p. 233-238.

(C) 2014 Wageningen Academic Publishers

The copyright holder has granted the permission for posting the article here.

Authors

C. K. Fenger, Thomas Tobin, P. J. Casey, Edward A. Roualdes, J. L. Langemeier, and D. M. Haines 


\title{
Bovine colostrum supplementation optimises earnings, performance and recovery in racing Thoroughbreds
}

\author{
C.K. Fenger ${ }^{1 *}$, T. Tobin ${ }^{2}$, P.J. Casey ${ }^{3}$, E.A. Roualdes ${ }^{4}$, J.L. Langemeier ${ }^{1}$ and D.M. Haines ${ }^{5,6}$ \\ 'Equine Integrated Medicine, PLC, 4904 Ironworks Rd., Georgetown, KY 40324, USA; ${ }^{2}$ Gluck Equine Research Center, University of Kentucky, \\ Lexington, KY 40546-0099, USA; ${ }^{3}$ Mont Liggins Charitable Trust, Research Centre in Reproductive Medicine, University of Auckland, P.O. \\ Box 110085, Auckland 1148, New Zealand; ${ }^{4}$ Department of Statistics, University of Kentucky, Lexington, KY 40504, USA; ${ }^{5}$ Department of \\ Veterinary Microbiology, Western College of Veterinary Medicine, University of Saskatchewan, 52 Campus Drive, Saskatoon, SK S7N5B4, \\ Canada; ${ }^{6}$ The Saskatoon Colostrum Co. Ltd., 30 Molaro Place, Saskatoon, SK S7K6A2, Canada; drfenger@hotmail.com
}

Received: 22 October 2014 / Accepted: 27 November 2014

RESEARCH ARTICLE

(c) 2014 Wageningen Academic Publishers

\begin{abstract}
Bovine colostrum $(\mathrm{BC})$ is the first milk produced by cows after calving and contains numerous beneficial substances for the immunity and development of the newborn calf. Because of the growth and immune factors in $\mathrm{BC}$, it has become an attractive supplement for use by athletes to support immunity and health during athletic performance. In order to evaluate the effects of oral BC supplementation on equine athletes, this study evaluated the earnings, performance, recovery and incidence of upper respiratory infections (URTI) in racing horses. The study design was a randomized cross-over racing performance study. 21 horses in race training were randomly assigned to train and compete with or without BC supplementation. After each horse competed in three races, it was crossed over to the other group, allowed a three week washout period, and then competed in three additional races. Horses in public training stables of 3 participating trainers were used. Race performance as determined by earnings, Bloodstock Research Information System (BRIS) speed figures, recovery as determined by number of days between races and incidence of upper respiratory tract disease was recorded. 11 horses completed the study. There was no effect of the order of $\mathrm{BC}$ supplementation on the measured variables. Horses on $\mathrm{BC}$ supplementation earned \$2,088 more purse money per race, than when unsupplemented $(P=0.016)$, and ran an average of 5 BRIS speed points higher $(P=0.03)$. Horses returned to racing on average 7.5 days faster (16.9 days vs 24.4 days, $P=0.048$ ). There were no URTI among the horses on $\mathrm{BC}$ supplementation and two infections while not on $\mathrm{BC}$ supplementation ( $\mathrm{z}$-test, $P=0.11$ ). Statistical analysis showed that horses recovered more quickly, earned three times more money and raced better as judged by BRIS scores while competing with BC supplementation. BC supplemented horses also experienced fewer URTI, although this effect was not significant.
\end{abstract}

Keywords: bovine colostrum, horse, performance, brisnet speed figures, upper respiratory tract infections

\section{Introduction}

Bovine colostrum (BC) is the first milk produced by cows after calving. BC contains immunoglobulins, other immune factors, growth factors and nutrients necessary for optimal immunity, support and long term performance of the neonatal calf. More recently, $\mathrm{BC}$ has been used as a nutritional supplement in humans for both immune support and for optimising athletic performance. In humans, BC has been shown to be effective in reducing the incidence of influenza (Cesarone et al., 2007), and upper respiratory tract infections (URTI) in children (Patel and Rana, 2006), and in athletes (Brinkworth and Buckley, 2003). In humans, BC has been found to improve endurance cycling performance (Coombes et al., 2002) and to enhance recovery and improve performance following sequential exercise sessions (Buckley et al., 2002; Shing et al., 2006). 
Horse racing is a highly competitive sport in which trainers and veterinarians strive to maintain their animals in optimal health. Dietary supplements that support immune responses and thereby optimise the health, welfare and athletic performance of horses are potentially of great benefit to the racing industry. While the effect of BC dietary supplementation may be related to reported increases in plasma immunoglobulin (IGF)-1 in humans (Mero et al., 1997), no such BC supplementation increases in IGF-1 occurs in horses associated with the BC supplementation protocol reported herein (Fenger et al., 2014).

Given these considerations, this study was undertaken to determine if $\mathrm{BC}$ has a beneficial effect on the incidence of URTI and athletic performance in Thoroughbred racehorses.

\section{Materials and methods}

\section{Study facilities and animals}

Privately owned Thoroughbred racehorses in race training in Central Kentucky were used throughout. Horses were housed and trained according to standard racing stable management procedures at a Thoroughbred training facility in Lexington, KY, USA. Horses were stabled in $3.7 \times 3.7$ $\mathrm{m}$ stalls bedded in straw. Training consisted of trotting approximately 1.6 to $2.4 \mathrm{~km}$ ( 1 to 1.5 miles) and galloping approximately 1.6 to $2.4 \mathrm{~km}$ (1 to 1.5 miles) either on the dirt racetrack or a turf exercise field five days a week, with about one breezing day (fast gallop approaching racing speeds for 0.6 to $1.0 \mathrm{~km} \mathrm{(3/8} \mathrm{to} \mathrm{5/8} \mathrm{mile)} \mathrm{per} \mathrm{week} \mathrm{and} \mathrm{one} \mathrm{day} \mathrm{of}$ walking only per week. Races were chosen according to the standard operating procedures of the 3 trainers involved. The study protocol was approved by the institutional animal care and use committee of Equine Integrated Medicine PLC and for all horses participating in the study, the owner or his or her designee (i.e. the trainer) provided informed consent.

\section{Bovine colostrum}

The colostrum used in this trial was produced under USDA and CFIA permits for commercial sale as a veterinary biologic. Excess colostrum was collected from dairies licensed to produce milk for human consumption and stored frozen $\left(-18^{\circ} \mathrm{C}\right)$ until processing. The colostrum was then thawed at room temperature until softened sufficiently to be pooled following pulverisation by an ice crusher, then pasteurised using a proprietary method (Saskatoon Colostrum Company, Ltd., Sasketchewan, Canada) developed to eliminate pathogenic contaminants while maintaining the integrity of the immune factors and spray dried. The spray dried colostrum was tested for retention of potency using an radial immunodiffusion method for IgG and for safety using standard microbiological methods for total plate counts, coliforms and Salmonella. The BC powder was then packaged by Animal Healthcare Products and Packaging, Inc. (Winchester, KY, USA) according to good manufacturing practices protocol before being shipped to the study location.

\section{Experimental design}

All racehorses in the three participating trainers' control were included in this cross-over study. Trainers were previously known to one of the investigators (CF) to be reliable and able to comply with the study procedures. Horses were randomly assigned to the $\mathrm{BC}$ supplementation first or no BC supplementation first groups. Horses in the treatment group received $100 \mathrm{~g} \mathrm{BC}$ daily as a feed top dress for at least weeks prior to the first race and until 3 races were completed, at which point they had a 3 week washout period, followed by three races without BC supplement. Horses in the no BC supplementation first group had three races, followed by at least a 3 week acclimation period on 100 g BC daily, which period continued until 3 races were completed. Horses which were claimed, sold or retired prior to completing the 6 race regimen were excluded from the performance data analysis. All horses were included in the URTI incidence data. Horses that completed the study were all 3 or 4 year olds, with 3 geldings, a male and 2 females in the no-treatment first group and four females and a gelding in the treatment first group.

Because of the differences in racing class, time of year, track surface and condition, the times or finish positions of the races were not considered to be useful for analysis. Racing performance was analysed based on the BRIS speed figure for each race; Bloodstock Research Information System (BRIS) speed figures were generated by an independent organisation (Bloodstock research Information System, Lexington, KY, USA; www.brisnet.com) and recorded for each of the six races for each horse. This standardised number represents a racing score independent of other factors. The amount of money earned for each race was similarly recorded. The total amount of money earned was averaged for each horse for the three races in each group. Recovery days between races were similarly averaged for all $\mathrm{BC}$ days and all non-BC days for each horse. Layoffs associated with injuries were excluded from the recovery data. Upper respiratory tract infections were defined as a nasal discharge and cough, which was diagnosed by endoscopy to be due to either lymphoid hyperplasia or guttural pouch infection.

\section{Data analysis}

The analysis of variance for a cross-over design, controlling for period, sequence, and horse, was fit to the data. Normality in each of the independent variables was determined by visual inspection of normality plots and Anderson-Darling test of normality, and analysed using a 
general linear model (Proc GLM; SAS Institute, Cary, NC, USA). When normality assumptions were not fulfilled, the data were transformed before analysis to appropriately meet model assumptions. Normality assumptions were met for the analysis of average BRIS speed figures, money earned in the treatment and control races, and for the square root transformed recovery days. F-tests were used to determine the presence of carryover and sequence effects. Incidence of URTI was analysed using a z-test.

\section{Results}

A total of 21 racehorses were originally enrolled in the study, with 11 horses completing the study. Seven horses were sold before completing 6 races and 3 were 2 year olds which never started during the study period. Because the horse sales and 2 year olds were independent of the assigned treatment, the 10 horses not contained in the analysis are considered missing at random and hence are unlikely to unduly bias the results presented here. Of the 11 horses completing the study, 2 horses were excluded from the recovery analysis owing to injuries, which affected the days off between races. An ex-post power study was performed on the cross-over design used to analyse these data. Across all response variables, a maximum of 10 horses was necessary for least $80 \%$ power, hence the 11 horses considered in this study is sufficient for average horse effects. Further studies, using a greater number of horses, would be required to find different effects of bovine colostrum across genders. However, gender stratification is shown in the figures to demonstrate that there appears to be no difference between genders.

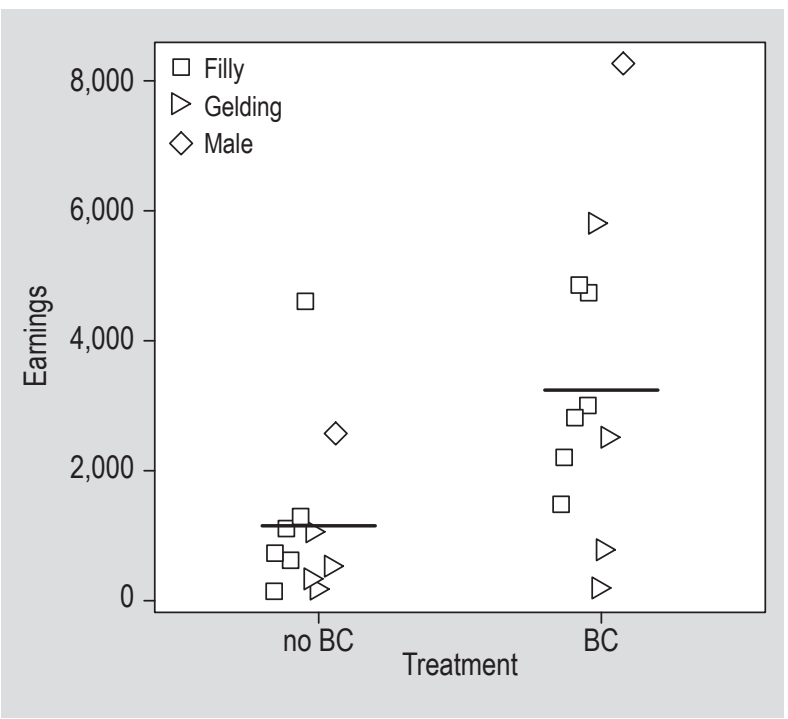

Figure 1. Average race earnings (US $\$, P=0.016$ ) for all horses with or without bovine colostrum (BC) supplementation (randomly jittered along the $\mathrm{x}$-axis for clarity). The horizontal bars across each treatment represents the least squares mean as predicted by the model fit to the data shown.
A standard linear, normal model was fit to the data using the appropriate analysis of variance model for a cross-over design, controlling for period, sequence, and horse. For each response variable we investigated departures from normality with both visual inspections and an Anderson-Darling test of normality. Neither earnings nor BRIS numbers deviated from the assumption of normality, however, Recovery (days between races) failed the normality test, so a square root transformation was performed before analysis. No response variable showed a sequence effect (earnings, $P=0.48$; BRIS numbers, $P=0.12$; recovery, $P=0.33$ ) or carry-over effect (earnings, $P=0.16$; BRIS numbers, $P=0.53$; recovery, $P=0.24$ ), thus suggesting our cross-over model is not inappropriately fit to these data. Earnings were higher $(P=0.016)$ with least squares means of $\$ 3,241$ for horses while receiving $B C$ supplementation compared to $\$ 1,153$ for horses while not on BC (Figure 1). The data also show an improvement in speed (BRIS number, $P=0.03$ ) with horses running a least squares mean of 67 while racing on $\mathrm{BC}$ and 62 when not on $\mathrm{BC}$ (Figure 2). Horses also returned to racing 7.5 days quicker ( 16.9 days vs 24.4 days, $P=0.048$ ) while supplemented with BC (Figure 3). In this study, no statistically significant effect of $B C$ supplementation could be identified on the incidence of URTI $(P=0.11)$, likely because of the small number of test animals and the relatively small incidence of overt URTI in this cohort.

\section{Discussion}

Our data demonstrated a clear improvement in athletic performance in horses receiving $\mathrm{BC}$ supplementation. Horses raced faster (higher BRIS speed figures) with

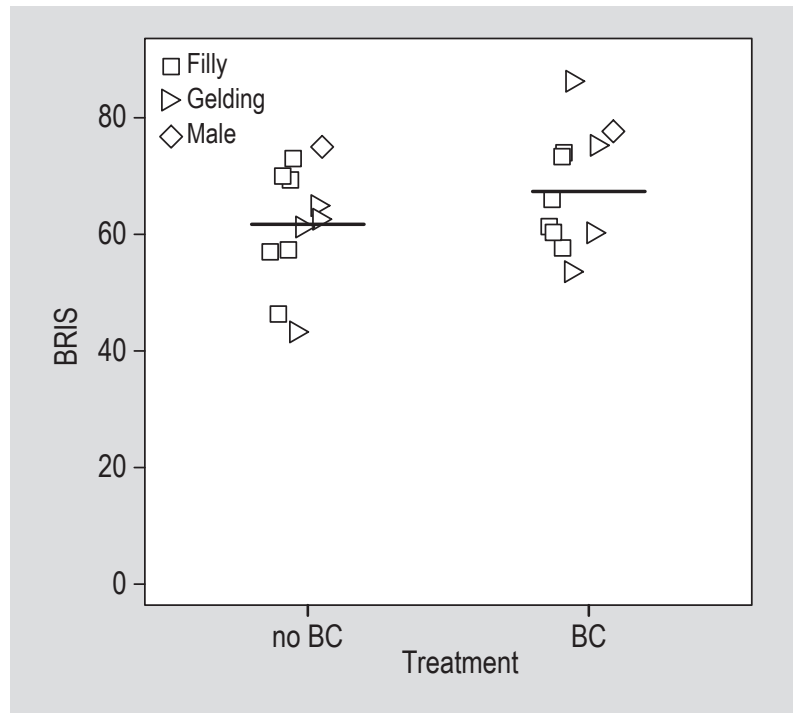

Figure 2. Average $B R I S$ speed ratings $(P=0.03)$ for all horses with or without bovine colostrum (BC) supplementation (randomly jittered along the $\mathrm{x}$-axis for clarity). The horizontal bars across each treatment represents the least squares mean as predicted by the model fit to the data shown. 


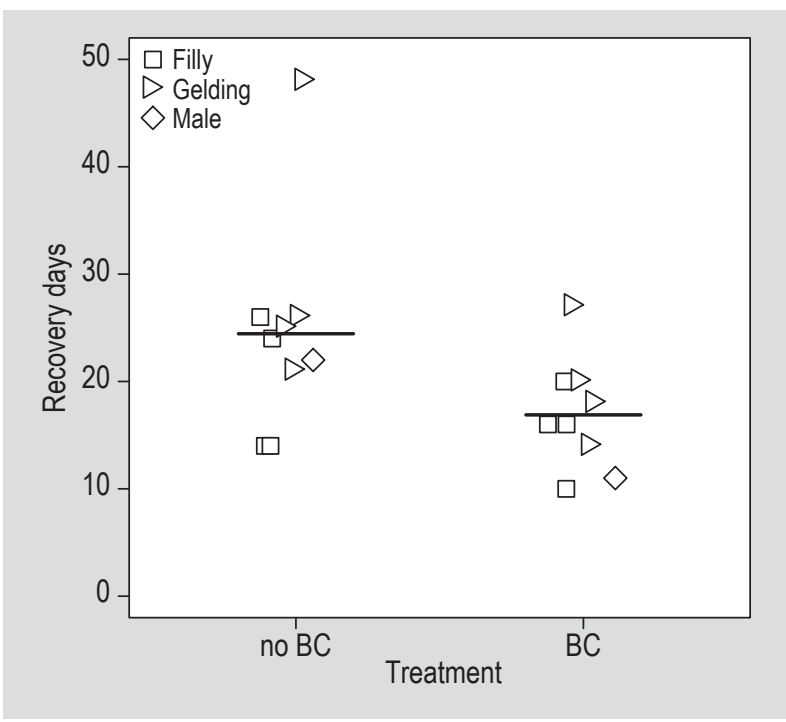

Figure 3. Average recovery days between races for the second and third races with or without bovine colostrum (BC) supplementation for all horses (randomly jittered along the $x$-axis for clarity). The horizontal bars across each treatment represents the least squares mean as predicted by the model fit to the data shown. Two horses were excluded from analysis because of illness or injury. Statistical analysis was performed on transformed data $(P=0.048)$, but non-transformed data are shown here.

aggregate greater earnings of $\$ 70,455$ over the course of this study while supplemented with BC. BRIS speed figures are calculated based on a computed algorithm which takes into account racetrack, surface, track condition, quality of the other horses in the race, and other factors contributing to the racing performance of a horse in a given day (www.brisnet.com). This is a performance metric which is comparable across all races on all days and is computed for all horses in North America. BRIS speed figures translate into approximately 1 point per 1-1.5 lengths in a race, with the value of a length decreasing as the race distance increases to accurately reflect racing effort. Therefore, the 5 BRIS speed figure improvement in racing performance in horses on BC supplementation reflects an approximately 5 length improvement in racing performance.

The performance effects of dietary supplementation with $\mathrm{BC}$ in human athletes is mixed. No improvement in performance was seen in a small double-blind study in healthy men and women undergoing aerobic and resistance training (Antonio et al., 2001), although there was an increase in lean body mass. Similarly, no performance effect was seen in endurance exercise in male and female hockey players (Hofman et al., 2002), rowing ability in elite female rowers (Brinkworth et al., 2002), or a single exercise-to-exhaustion test (Buckley et al., 2002). On the other hand, a small improvement in a $2 \mathrm{~h}$ endurance time trial in cyclists after an 8-week supplementation period has been reported (Coombes et al., 2002), and a recent study found increased leg press strength when older adults were supplemented with BC (Duff et al., 2014).

The mechanism of athletic performance improvement in humans has not been determined, but has been suggested to be associated with an increase in serum IGF-1 concentration (Mero et al., 1997). However, the magnitude of the increase of IGF-1 seen in the Mero study was small, did not exceed the normal range, and has not been repeatable by other investigators (Buckley et al., 2002; Coombes et al., 2002; Duff et al., 2014). Similarly to these latter studies, there is no increase in IGF-1 in Thoroughbred race horses associated with BC supplementation (Fenger et al., 2014). This indicates that any improvement in performance seen with $\mathrm{BC}$ in these horses was independent of an IGF-1 effect.

One possible mechanism could be related to other cytokines in $B C$, such as interleukin (IL)-1 $\beta$, IL-6, tumour necrosis factor alpha (TNF- $\alpha$ ), interferon-gamma (INF- $\gamma$ ) and IL-1 receptor antagonist (IL-1ra), any of which may have an effect on athletic performance. While most cytokines would typically be degraded in the digestive tract, it has been suggested that cytokines in $\mathrm{BC}$ may be protected by some unidentified mechanism, resulting in their survival through the digestive process and absorption into the circulation (Davison, 2012). The role of inflammatory cytokines, such as IL-1 $\beta$, IFN- $\gamma$, IL- 6 and TNF- $\alpha$ during exercise is to send critical signals to exercising muscles that reactive oxygen species and other indicators of stress are forthcoming. These critical signals set off a cascade of reactions mediated by nuclear factor kappa-beta (NF-k $\beta$ ), which moderates adaptation to exercise (Radak et al., 2013). If these cytokines are absorbed from the $\mathrm{BC}$, then this adaptation may be the mechanism of the improved performance during exercise.

Post-exercise recovery was improved with BC supplementation in our subjects. Horses were able to race back 7.5 days quicker, reflecting a shorter recovery time after a maximal racing effort in our study. This is a similar finding to studies in humans. In an 8-week, double-blinded, placebocontrolled study, BC supplementation did not improve performance on an exercise-to-exhaustion test. However, it did improve performance on a repeat test 20 min later (Buckley et al., 2002). In cyclists performing high intensity exercise tests with short recovery times, $\mathrm{BC}$ supplementation also benefitted recovery (Shing et al., 2006). The improved recovery may be the result of a reduction in oxidative stress in skeletal muscle during exercise while supplemented with BC (Appukutty et al., 2013).

In our study, there was no difference in incidence of overt URTI in horses while BC supplemented. However, this was likely due to an overall low rate of overt URTI in this cohort. In humans, $\mathrm{BC}$ supplementation decreased the incidence of influenza (Cesarone et al., 2007), URTI in 
children (Patel and Rana, 2006), and in athletes (Brinkworth and Buckley, 2003). Intense exercise training is well known to cause immunosuppression in humans (Smith, 2003) and horses (Davis et al., 2007) which can manifest as URTI. This has been suggested to result from a suppression of TH-2 cell-mediated immunity by an exercise related increase in the TH-1 immune response (Smith, 2003), and an increase in natural killer (NK) cells (Wong et al., 2014). In human studies, BC supplementation has multiple immune system effects in athletes, including increasing salivary immunoglobulin A concentrations in runners (Crooks et al., 2006), improvement in the recovery of neutrophil function and maintenance of salivary lysozyme concentrations in cyclists (Davison and Diment, 2010), and preventing the TH-2 immune suppression associated with exercise in cyclists (Shing et al., 2007). While our URTI data did not attain statistical significance, the overall incidence of overt URTI was low in our group. This was likely the result of the requirement of the crossover model for 6 or 7 sequential races. Often, younger horses, which are more susceptible to overt URTI, fail to make this number of races sequentially without substantial layoffs. Nonetheless, the only overt URTI were in the horses during the non-BC supplemented period, and it is likely that subclinical infection also occurs, which may have detrimental performance effects. The mechanism of decreased incidence of URTI in athletes supplemented with $\mathrm{BC}$ is likely associated with an immune modifying effect of the $\mathrm{BC}$ supplementation, presumably offsetting the transient immunosuppression associated with intense exercise. An immune modifying effect may have influenced performance and recovery in this study, although this cannot be unequivocally shown with these data.

The horse is physiologically unique in that as soon as it begins to exercise it fails to fully oxygenate its blood (Fenger et al., 2000), suffering from 'exercise induced hypoxemia'. The stroke volume of blood travels so rapidly across the pulmonary circulation that there is insufficient time for complete oxygenation across the thin alveolar-capillary membrane. Inflammation in the airways as may be caused by subclinical infection acts to exacerbate 'exercise induced hypoxemia' in horses, by thickening this alveolar-capillary membrane. Demonstrating this phenomenon, Sánchez et al. (2005) showed that the faster a horse runs the greater the oxygen deficit in the blood delivered to the muscles, and that this deficit is exacerbated by airway inflammation. Any intervention that reduces airway inflammation may be expected to immediately and proportionally improve oxygen delivery to the exercising musculature and thus equivalently improve racing performance, and may have been the mechanism of the performance benefit seen in the present study.

In conclusion, $\mathrm{BC}$ can be supplemented to racehorses for the purpose of optimising athletic performance and recovery of the animal. Further studies to elucidate the optimal dose, extent and specific mechanisms of immune modifying effect of $\mathrm{BC}$ in exercising horses are warranted.

\section{Acknowledgements}

We thank The Saskatoon Colostrum Company, Ltd. for providing PETerna ${ }^{\mathrm{Tx}}$, and Animal Healthcare Products and Packaging, Inc. for providing funding for this project. Published as Kentucky Agricultural Experiment Station Article 14-14-052 with approval of the Dean and Director, College of Agriculture and Kentucky Agricultural Experimental Station.

\section{References}

Antonio, J., Sanders, M. and Van Gammeren, D., 2001. The effects of bovine colostrum supplementation on body composition and exercise performance in active men and women. Nutrition 17: 243-247.

Appukutty, M., Radhakrishnan, A.K., Ramasamy, K., Ramasamy, R., Abdul Majeed, A.B., Noor, M.I., Safii, N.S., Koon, P.B., Chinna, K. and Haleagrahara, N., 2013. Colostrum supplementation protects against exercise-induced oxidative stress in skeletal muscle in mice. BMC Research Notes 5: 649.

Brinkworth, G. and Buckley, J., 2003. Concentrated bovine colostrum protein supplementation reduces the incidence of self-reported symptoms of upper respiratory tract infection in adult males. European Journal of Nutrition 42: 228-232.

Brinkworth, G.D., Buckley, J.D., Bourdon, P.C., Gulbin, J.P. and David, A., 2002. Oral bovine colostrum supplementation enhances buffer capacity but not rowing performance in elite female rowers. International Journal of Sport Nutrition Exercise Metabolism 12: 349-365.

Buckley, J., Abbott, M., Brinkworth, G. and Whyte, P., 2002. Bovine colostrum supplementation during endurance running training improves recovery, but not performance. Journal of Science and Medicine in Sport 5: 65-79.

Cesarone, M., Belcaro, G., Di Renzo, A., Dugall, M., Cacchio, M., Ruffini, I., Pellegrini, L., Del Boccio, G., Fano, F., Ledda, A., Bottari, A., Ricci, A., Stuard, S. and Vinciguerra, G., 2007. Prevention of influenza episodes with colostrum compared with vaccination in healthy and high-risk cardiovascular subjects: the epidemiologic study in San Valentino. Clinical and Applied Thrombosis/ Hemostasis 13: 130-136.

Coombes, J., Conacher, M., Austen, S. and Marshall, P., 2002. Dose effects of oral bovine colostrum on physical work capacity in cyclists. Medicine and Science in Sports and Exercise 34: 1184-1188.

Crooks, C., Wall, C., Cross, M. and Rutherfurd-Markwick, K., 2006. The effect of bovine colostrum supplementation on salivary IgA in distance runners. International Journal of Sport Nutrition and Exercise Metabolism 16: 47-64.

Davis, M., Williams, C., Meinkoth, J., Malayer, J., Royer, C., Williamson, K. and McKenzie, E., 2007. Influx of neutrophils and persistence of cytokine expression in airways of horses after performing exercise while breathing cold air. American Journal of Veterinary Research 68: 185-189. 


\section{C.K. Fenger et al.}

Davison, G. and Diment, B., 2010. Bovine colostrum supplementation attenuates the decrease of salivary lysozyme and enhances the recovery of neutrophil function after prolonged exercise. British Journal of Nutrition 103: 1425-1432.

Davison, G., 2012. Bovine colostrum and immune function after exercise. Medicine and Sport Science 59: 62-69.

Duff, W., Chilibeck, P., Rooke, J., Kaviani, M., Krentz, J.R. and Haines, D.M., 2014. The effect of bovine colostrum supplementation in older adults during resistance training. International Journal of Sport Nutrition Exercise Metabolism 24: 276-285.

Fenger, C.K., McKeever, K.H., Hinchcliff, K.W. and Kohn, C.W., 2000. Determinants of oxygen delivery and hemoglobin saturation during incremental exercise in horses. American Journal of Veterinary Research 61: 1325-1332.

Fenger, C.K., Tobin, T., Casey, P.J., Langemeier, J.L. and Haines, D.M., 2014. Bovine colostrum supplementation does not influence serum IGF-1 in horses in race training. Journal of Equine Veterinary Science 34: 1025-1027.

Hofman, Z., Smeets, R., Verlaan, G., Lugt, R. and Verstappen, P., 2002. The effect of bovine colostrum supplementation on exercise performance in elite field hockey players. International Journal of Sport Nutrition and Exercise Metabolism 12: 461-469.

Mero, A., Miikkulainen, H., Riski, J., Pakkanen, R., Aalto, J. and Takala, T., 1997. Effects of bovine colostrum supplementation on serum IGF-I, IgG, hormone, and saliva IgA during training. Journal of Applied Physiology 83: 1144-1151.
Patel, K. and Rana, R., 2006. Pedimune in recurrent respiratory infection and diarrhoea - the Indian experience - the pride study. Indian Journal of Pediatrics 73: 585-591.

Radak, Z., Zhao, Z., Koltai, E., Ohno, H. and Atalay, M., 2013. Oxygen consumption and usage during physical exercise: the balance between oxidative stress and ROS-dependent adaptive signaling. Antioxidants and Redox Signaling 18: 1208-1246.

Sánchez, A., Couëtil, L.L., Ward, M.P. and Clark, S.P., 2005. Effect of airway disease on blood gas exchange in racehorses. Journal of Veterinary Internal Medicine 19: 87-92.

Shing, C.M., Jenkins, D.G., Stevenson, L. and Coombes, J.S., 2006. The influence of bovine colostrum supplementation on exercise performance in highly trained cyclists. British Journal of Sports Medicine 40: 797-801.

Shing, C.M., Peake, J., Suzuki, K., Okutsu, M., Pereira, R., Stevenson, L., Jenkins, D.G. and Coombes, J.S., 2007. Effects of bovine colostrum supplementation on immune variables in highly trained cyclists. Journal of Applied Physiology 102: 1113-1122.

Smith, L., 2003. Overtraining, excessive exercise, and altered immunity: is this a T helper-1 versus T helper-2 lymphocyte response? Sports Medicine 33: 347-364.

Wong, E.B., Mallet, J.F., Duarteb, J., Matar, C. and Ritz, B.W., 2014. Bovine colostrum enhances natural killer cell activity and immune response in a mouse model of influenza infection and mediates intestinal immunity through toll-like receptors 2 and 4 . Nutrition Research 34: 318-325. 